\title{
The Index of Transversally Elliptic Operators on Locally Homogeneous Spaces of Finite Volume
}

\author{
JefFrey FoX ${ }^{1}$ \& Peter Haskell ${ }^{2}$
}

\section{Introduction}

There is a well-developed index theory of elliptic operators on compact manifolds. On noncompact manifolds a variety of approaches to index theory of elliptic operators has yielded interesting information under various assumptions. On a class of noncompact, but finite-volume, locally homogeneous spaces, elliptic differential operators descended from invariant operators on the associated homogeneous spaces can be used to define Fredholm operators with interesting indices. In general, to define the Fredholm operator one must restrict the elliptic operator to the "discrete summand" of a spectral decomposition determined by the Lie group used to define the locally homogeneous space. There is a large literature on this subject. A concise discussion of the aspects relevant to our paper appears in [Mo].

There is also an index theory of operators elliptic in directions transverse to group actions or to foliations, especially on compact manifolds [At; NZ; $\mathrm{Si}$; Ve; Co; CS; HS]. In [FH2] we studied operators $T$ invariant under and elliptic in directions transverse to locally free actions of noncompact Lie groups $G$ on compact manifolds. Such an operator is not generally Fredholm, but each irreducible $G$-representation occurs with finite multiplicity in the kernels of the operator and its adjoint. In [FH2] we showed how to use the indices of elliptic operators on compact manifolds to calculate, for some irreducible $G$-representations $\beta$, the difference: multiplicity of $\beta$ in $\operatorname{kernel}(T)$ minus multiplicity of $\beta$ in $\operatorname{kernel}\left(T^{*}\right)$.

In the present paper we extend the above results to certain noncompact locally homogeneous settings. We give in Section 1 the precise assumptions under which we work, as well as an indication of the variety and complexity of examples that occur. In this introduction we describe our setting less carefully as follows. Let $G_{1}$ and $G_{2}$ be noncompact, connected, semisimple Lie groups. Assume that $H$ is a compact subgroup of $G_{2}$ and that $\Gamma$ is a lattice

Received November 17, 1992.

${ }^{1}$ Work supported by the National Science Foundation under grant no. DMS-8903472.

2 Work supported by the National Science Foundation under grant nos. DMS-8901436 and DMS-9204275.

Michigan Math. J. 41 (1994). 
in $G_{1} \times G_{2}$. Let $K_{1}$ be the maximal compact subgroup of $G_{1}$. Let $T$ be a firstorder differential operator on $\left(G_{1} \times\left(H \backslash G_{2}\right)\right) / \Gamma$ that is invariant under and elliptic in directions transverse to the left $G_{1}$-action. $T$ must also be descended from a $\left(G_{1} \times G_{2}\right)$-invariant operator on $G_{1} \times\left(H \backslash G_{2}\right)$. (Note that $G_{1}$-orbits may be dense in $\left(G_{1} \times\left(H \backslash G_{2}\right)\right) / \Gamma$ and that $\left(G_{1} \times\left(H \backslash G_{2}\right)\right) / \Gamma$ may be noncompact.) $T$ acts on sections of locally homogeneous vector bundles. The underlying role of the Lie group $G_{1} \times G_{2}$ in these definitions determines a decomposition of the Hilbert spaces of $L^{2}$ sections of these bundles into discrete and continuous summands. $T$ respects this decomposition, and in the rest of this paragraph we use $T$ to denote the restriction of $T$ to the discrete summand. Each irreducible $G_{1}$-representation occurs with finite multiplicity in $\operatorname{kernel}(T)$ and $\operatorname{kernel}\left(T^{*}\right)$. For certain of these representations $\beta$ we describe an elliptic operator on $\left(K_{1} \times H\right) \backslash\left(G_{1} \times G_{2}\right) / \Gamma$ whose Fredholm index (in the sense appropriate to noncompact locally homogeneous spaces that is mentioned in the first paragraph) equals the difference: multiplicity of $\beta$ in $\operatorname{kernel}(T)$ minus multiplicity of $\beta$ in $\operatorname{kernel}\left(T^{*}\right)$.

In outline, our methods are as follows. A Dirac operator on $K_{1} \backslash G_{1}$ defines an element of $K K\left(\mathbb{C}, C^{*} G_{1}\right)$. The operator $T$ defines two different cycles representing the same element of $K K\left(C^{*} G_{1}, \mathbb{C}\right)$. We use the two different cycles to do two different calculations of the same Kasparov product (of the Dirac operator and the transversally elliptic operator) over $C^{*} G_{1}$. The Kasparov products lie in $K K(\mathbb{C}, \mathbb{C}) \cong \mathbb{Z}$. One calculation leads naturally to the index of an elliptic operator. The other, when the Dirac operator realizes a discrete series representation $\beta$, leads to the multiplicity expression.

Our reasoning relies heavily on the analysis appearing in [BG], [CM], [K2], and [Mo]. Sometimes-when the details of calculations are the same as those used in [FH1] or [FH2] - we substitute a reference to the previous papers for the details. The relation of our work to [Co], [CS], and [HS] is more complicated. In spirit our work is based upon theirs: longitudinally and transversally elliptic operators define $K K$ classes whose products are reprepresented by interesting elliptic operators. However, the foliation algebras in the papers mentioned above involve algebras of continuous functions, and such algebras in our setting would not respect the spectral decompositions we use. Our contribution to the index theory of transversally elliptic operators lies in adapting analysis arising in representation theory to prove an index theorem for transversally elliptic operators on a class of noncompact locally homogeneous spaces.

\section{Assumptions}

In this section we state the assumptions that hold for the rest of the paper, and we indicate a source of examples satisfying these assumptions.

Let $G=G_{1} \times G_{2}$ be a product of linear, connected, semisimple Lie groups, and let $\Gamma$ be a torsion-free discrete subgroup having finite covolume in $G$. (More generally, assume that $G$ and $\Gamma$ satisfy the assumptions of Section 2.1 
of [BG].) Let $H$ be a compact subgroup of $G_{2}$. We think of $H$ as a subgroup of $G$ by identifying it with $\left\{e_{1}\right\} \times H$.

Let $T$ be a first-order differential operator on $H \backslash G / \Gamma$ that maps sections of a locally homogeneous bundle $\mathbf{F}_{0}$ to sections of a locally homogeneous bundle $\mathbf{F}_{1}$. (A locally homogeneous bundle $\mathbf{F}$ arises from a right unitary representation of $H$ on a vector space $F$ via the construction $\mathbf{F}=F \times{ }_{H} G / \Gamma \rightarrow$ $H \backslash G / \Gamma$.) Assume further that $T$ is the descended version of a first-order $G$ invariant differential operator $\tilde{T}$ on $H \backslash G$ from sections of $F_{0} \times_{H} G$ to sections of $F_{1} \times{ }_{H} G$.

The natural left action of $G_{1}$ on $H \backslash G$ determines actions of $G_{1}$ on $F_{i} \times{ }_{H} G$ and on $F_{i} \times_{H} G / \Gamma$. We assume that $T$ is $G_{1}$-invariant and that $T$ is transversally elliptic relative to the $G_{1}$-action. Using Haar measure to place a measure on $H \backslash G / \Gamma$ in the standard way, we can define Hilbert spaces of $L^{2}$ sections of $\mathbf{F}_{i}$ on which the actions of $G_{1}$ are unitary.

ExAMPLE. Let $G_{1}$ and $G_{2}$ be $S L(2, \mathbb{R})$. Let $H$ be $S O(2)$. Let $\Gamma$ be a torsionfree subgroup of finite index in $S L(2, \mathbb{Z}[\sqrt{2}]) . \Gamma$ is imbedded in $G$ via the map $\left[a_{i j}+b_{i j} \sqrt{2}\right] \rightarrow\left(\left[a_{i j}+b_{i j} \sqrt{2}\right],\left[a_{i j}-b_{i j} \sqrt{2}\right]\right) . G_{1}$-orbits are dense in $H \backslash G / \Gamma$. A discussion of this example and of the method called restriction of scalars, with which many similar examples can be constructed, appears in [Zi]. In our example the operator $\tilde{T}$ is the tensor product of the identity operator on functions on the first factor of $S L(2, \mathbb{R})$ with the Dirac operator on spinors over $S O(2) \backslash S L(2, \mathbb{R})$.

\section{Locally Homogeneous Constructions}

In this section we describe some constructions and arguments that work on locally homogeneous spaces of the type we consider.

Using Haar measure on $G$, one can define $L^{2}(G / \Gamma)$. Translation defines a unitary representation of $G$ on $L^{2}(G / \Gamma)$. This representation decomposes into the direct sum of two unitary representations, called the discrete and continuous summands:

$$
L^{2}(G / \Gamma)=L_{d}^{2}(G / \Gamma) \oplus L_{c}^{2}(G / \Gamma) .
$$

If $K$ is a compact subgroup of $G$ ( $K$ need not be the maximal compact subgroup), and if there is a right unitary representation of $K$ on a vector space $W$, we denote the corresponding homogeneous bundle by

$$
\tilde{\mathbf{w}} \rightarrow K \backslash G
$$

and the corresponding locally homogeneous bundle by

$$
\mathbf{W} \rightarrow K \backslash G / \Gamma \text {. }
$$

$W$ may be graded, in which case $\tilde{\mathbf{W}}$ and $\mathbf{W}$ are also. We denote by $E_{\tilde{\mathbf{W}}}$ the Hilbert $C^{*} G$-module defined by sections of $\tilde{\mathbf{W}}$ (see [K2]). We denote by $L^{2}(\mathbf{W})$ the Hilbert space of $L^{2}$ sections of $\mathbf{W}$. 
Proposition 2.1 [FH1]. $\quad L^{2}(\mathbf{W}) \cong E_{\tilde{\mathbf{W}}} \otimes_{C^{*} G} L^{2}(G / \Gamma) \cong\left(W \otimes L^{2}(G / \Gamma)\right)^{K}$, by which we mean the set of $K$-invariant elements of $W \otimes L^{2}(G / \Gamma)$. If we replace $L^{2}(G / \Gamma)$ in the preceding sentence by $L_{d}^{2}(G / \Gamma)\left(\right.$ resp. $\left.L_{c}^{2}(G / \Gamma)\right)$, we get a Hilbert space that we denote by $L_{d}^{2}(\mathrm{~W})\left(\right.$ resp. $\left.L_{c}^{2}(\mathrm{~W})\right)$.

Proposition 2.2. A G-invariant, properly supported, pseudodifferential operator of nonpositive order acting on sections of $\tilde{\mathbf{W}}$ descends to define an operator on $L^{2}(\mathbf{W})$ that is block diagonal with respect to the decomposition $L^{2}(\mathbf{W})=L_{d}^{2}(\mathbf{W}) \oplus L_{c}^{2}(\mathbf{W})$. If the order of the pseudodifferential operator is negative, the discrete block of the descended operator is compact.

Proof. See [CM] and [K2]. The proof of the last sentence depends on a result of [BG] and is given in detail in [FH1, Proof of Prop. 3.15]. The gist of the argument involves relating the kernel representing (a high enough power of) the negative-order pseudodifferential operator to a continuous function on $G$, the action of which on $L_{d}^{2}(G / \Gamma)$ [BG] shows to be compact.

Proposition 2.3 [CM]. A G-invariant, properly supported, elliptic pseudodifferential operator $\tilde{L}$ of positive order acting on sections of $\tilde{\mathbf{W}}$ has a parametrix $\tilde{S}$ that is a G-invariant, properly supported pseudodifferential operator of negative order. $\tilde{L} \circ \tilde{S}-I$ and $\tilde{S} \circ \tilde{L}-I$ are G-invariant, properly supported smoothing operators.

COROLLARY 2.4. Let $L$ and $S$ be the descended operators on $L^{2}(\mathrm{~W})$ associated with the $\tilde{L}$ and $\tilde{S}$ of the preceding proposition. Then the restrictions of $L \circ S-I$ and $S \circ L-I$ to $L_{d}^{2}(\mathbf{W})$ define compact operators.

Proof. The argument proving [FH1, Prop. 3.15] applies.

COROLlaRY 2.5. In the setting of the preceding corollary, assume further that $L$ has a bounded inverse $L^{-1}$. Then the restriction of $L^{-1}$ to $L_{d}^{2}(\mathbf{W})$ is compact.

Proof. We restrict to $L_{d}^{2}(\mathrm{~W})$ where $S$ is compact. $L \circ S-I$ is compact, and $L \circ L^{-1}-I=0$. Thus $L \circ\left(S-L^{-1}\right)$ is compact, and so is $S-L^{-1}$.

Let $\mathcal{U}\left(\mathfrak{g}^{\mathbb{C}}\right)$ denote the universal enveloping algebra for the complexification of the Lie algebra of $G$. Associated to each $G$-invariant differential operator on sections of $\tilde{\mathbf{W}} \rightarrow K \backslash G$ is an element $\sum_{i} A_{i} \otimes X_{i}$ of $\left(\operatorname{Hom}(W, W) \otimes u\left(\mathrm{~g}^{\mathbb{C}}\right)\right)^{K}$ (see e.g. [Mo]). (If we replace $\operatorname{Hom}(W, W)$ by $\operatorname{Hom}\left(W_{i}, W_{j}\right)$ then the same framework applies.) Associated to the representation of $G$ on $L^{2}(G / \Gamma)=$ $L_{d}^{2}(G / \Gamma) \oplus L_{c}^{2}(G / \Gamma)$ is what is known as the set of $C^{\infty}$ vectors of this representation, $L^{2}(G / \Gamma)_{\infty}=L_{d}^{2}(G / \Gamma)_{\infty} \oplus L_{c}^{2}(G / \Gamma)_{\infty}$. The $G$-representation defines derived representations $\rho=\rho_{d} \oplus \rho_{c}$ of $\mathcal{U}\left(\mathfrak{g}^{\mathbb{C}}\right)$ on

$$
L^{2}(G / \Gamma)_{\infty}=L_{d}^{2}(G / \Gamma)_{\infty} \oplus L_{c}^{2}(G / \Gamma)_{\infty}
$$

There is an action of $\left(\operatorname{Hom}(W, W) \otimes \mathcal{U}\left(\mathfrak{g}^{\mathbb{C}}\right)\right)^{K}$ on $L^{2}(\mathbf{W}) \cong\left(W \otimes L^{2}(G / \Gamma)_{\infty}\right)^{K}$ via 


$$
\sum_{i} A_{i} \otimes X_{i} \mapsto \sum_{i} A_{i} \otimes \rho\left(X_{i}\right) .
$$

This action and the following results respect the decomposition into discrete and continuous parts.

Proposition 2.6 [Mo]. If $\sum_{i} A_{i} \otimes X_{i}$ is elliptic of positive order and if $\sum_{i} A_{i} \otimes \rho\left(X_{i}\right)$ is symmetric on $\left(W \otimes L^{2}(G / \Gamma)_{\infty}\right)^{K}$, then $\Sigma_{i} A_{i} \otimes \rho\left(X_{i}\right)$ is essentially self-adjoint on $L^{2}(\mathrm{~W})$. If $\sum_{i} A_{i} \otimes \rho\left(X_{i}\right)$ has eigenvectors, they are smooth and thus lie in $\left(W \otimes L^{2}(G / \Gamma)_{\infty}\right)^{K}$.

COROLLARY 2.7. Let $\sum_{i} A_{i} \otimes X_{i}$ be an invertible positive-order operator of the type described in the preceding proposition. Then $\left(W \otimes L_{d}^{2}(G / \Gamma)\right)^{K}$ has a basis consisting of eigenvectors of $\sum_{i} A_{i} \otimes \rho_{d}\left(X_{i}\right)$. Each eigenvector lies in $\left(W \otimes L_{d}^{2}(G / \Gamma)_{\infty}\right)^{K}$. Each eigenspace is finite-dimensional.

Proof. This is a consequence of Proposition 2.6, Corollary 2.5, and the spectral theory of compact self-adjoint operators.

Proposition 2.8 (see [Mo]). $\quad \sum_{i} A_{i} \otimes X_{i} \in\left(\operatorname{Hom}(W, W) \otimes \mathcal{U}\left(\mathrm{g}^{\mathbb{C}}\right)\right)^{K}$ has a "formal adjoint" $\sum_{i} A_{i}^{*} \otimes X_{i}^{*} \in\left(\operatorname{Hom}(W, W) \otimes \mathcal{U}\left(\mathrm{g}^{\mathbb{C}}\right)\right)^{K}$. Here $A_{i}^{*}$ is the usual adjoint of $A_{i}$, and $X_{i}^{*}$ is defined by extending $X^{*}=-\bar{X}$ from $\mathrm{g}^{\mathbb{C}}$ to $\mathrm{U}\left(\mathrm{g}^{\mathbb{C}}\right)$.

REMARK 2.9. One can check that for $u, v \in\left(W \otimes L^{2}(G / \Gamma)_{\infty}\right)^{K}$, the inner product for $L^{2}(\mathrm{~W})$ satisfies

$$
\left\langle\left(\sum_{i} A_{i} \otimes \rho\left(X_{i}\right)\right) u, v\right\rangle=\left\langle u,\left(\Sigma_{i} A_{i}^{*} \otimes \rho\left(X_{i}^{*}\right)\right) v\right\rangle .
$$

Proposition 2.10. Let $\sum_{i} A_{i} \otimes X_{i}$ be as in Corollary 2.7. Assume that $\Sigma_{i} B_{i} \otimes \rho_{d}\left(X_{i}\right)$ is defined on and symmetric on $\left(W \otimes L_{d}^{2}(G / \Gamma)_{\infty}\right)^{K}$ and that it commutes with $\Sigma_{i} A_{i} \otimes \rho_{d}\left(X_{i}\right)$ on $\left(W \otimes L_{d}^{2}(G / \Gamma)_{\infty}\right)$. Then $\sum_{i} B_{i} \otimes \rho_{d}\left(X_{i}\right)$ is essentially self-adjoint.

Proof. $\Sigma_{i} B_{i} \otimes \rho_{d}\left(X_{i}\right)$ is symmetric on each eigenspace for $\Sigma_{i} A_{i} \otimes \rho_{d}\left(X_{i}\right)$, so the finite-dimensional spectral theorem provides a dense set of analytic vectors for $\sum_{i} B_{i} \otimes \rho_{d}\left(X_{i}\right)$. Nelson's analytic vector theorem [RS] implies that $\sum_{i} B_{i} \otimes \rho_{d}\left(X_{i}\right)$ is essentially self-adjoint.

\section{The Cycle Defined by a Transversally Elliptic Operator}

In this section we use the transversally elliptic operator introduced in Section 1 to construct a Kasparov $\left(C^{*} G_{1}, \mathbb{C}\right)$-bimodule and thus an element of $K K\left(C^{*} G_{1}, \mathbb{C}\right)$.

Let $T$ be the transversally elliptic operator of Section 1 . Following Section 2, we associate to $T$ an element $\Sigma_{i} A_{i} \otimes X_{i}$ of $\left(\operatorname{Hom}\left(F_{0}, F_{1}\right) \otimes \mathcal{U}\left(\mathrm{g}^{\mathbb{C}}\right)\right)^{H}$. As in Proposition 2.8, there is a "formal adjoint"

$$
\sum_{i} A_{i}^{*} \otimes X_{i}^{*} \in\left(\operatorname{Hom}\left(F_{1}, F_{0}\right) \otimes \mathcal{U}\left(\mathrm{g}^{\mathbb{C}}\right)\right)^{H} .
$$

Let $\rho=\rho_{d} \oplus \rho_{c}$ denote the derived representation of $\mathcal{U}\left(\mathrm{g}^{\mathbb{C}}\right)$ on $L^{2}(G / \Gamma)_{\infty}=$ $L_{d}^{2}(G / \Gamma)_{\infty} \oplus L_{c}^{2}(G / \Gamma)_{\infty}$. Let the vector bundle $\mathbf{F}$ be graded by $\mathbf{F}=\mathbf{F}_{0} \oplus \mathbf{F}_{1}$. 
Notation 3.1. Let $J$ denote the degree-1 operator on $\left(F \otimes L_{d}^{2}(G / \Gamma)_{\infty}\right)^{H}$ defined by $\sum_{i} A_{i} \otimes \rho_{d}\left(X_{i}\right)$ in the lower left corner and $\Sigma_{i} A_{i}^{*} \otimes \rho_{d}\left(X_{i}^{*}\right)$ in the upper right corner.

Definition 3.2 (see [War, p. 267]). Let $\left\{Z_{j}\right\}$ be a basis for the Lie algebra $\mathrm{g}_{1}$ of $G_{1}$. Then $1-\sum_{j} Z_{j}^{2} \in \mathcal{U}\left(\mathrm{g}^{\mathbb{C}}\right)$. We denote $1-\sum_{j} Z_{j}^{2}$ by $1-\Delta_{1}$.

REMARK 3.3. Under the action of $G_{1}$ on $\mathbf{F}, 1-\Delta_{1}$ defines a second-order differential operator on sections of $\mathbf{F}$ that is descended from a $G$-invariant operator on sections of $\tilde{\mathbf{F}}$.

Notation 3.4. By a slight abuse of notation, we let $1-\Delta_{1}$ denote the associated element of $\left(\operatorname{Hom}(F, F) \otimes \mathcal{U}\left(g^{\mathbb{C}}\right)\right)^{H}$ and let $\rho\left(1-\Delta_{1}\right)$ denote the associated operator on $\left(F \otimes L^{2}(G / \Gamma)_{\infty}\right)^{H}$.

Proposition 3.5. $\rho\left(1-\Delta_{1}\right)+J^{2}$ defines a second-order elliptic operator that is symmetric on $\left(F \otimes L^{2}(G / \Gamma)_{\infty}\right)^{H}$ and thus essentially self-adjoint, and bounded below by 1 , on $L^{2}(\mathbf{F})$.

Proof. This follows from a calculation (see e.g. [War, pp. 268-269]) and Proposition 2.6.

Proposition 3.6. The closure of $\mathfrak{J}$, which we will also denote by $\mathfrak{J}$, defines a self-adjoint operator on $L_{d}^{2}(\mathbf{F})$.

Proof. Apply Proposition 2.10 to $\mathfrak{J}$ and the elliptic operator of Proposition 3.5.

Notation 3.7. The functional calculus permits us to define $J \cdot\left(1+J^{2}\right)^{-1 / 2} \in$ $\mathcal{L}\left(L_{d}^{2}(\mathbf{F})\right)$.

THEOREM 3.8. The unitary representation of $G_{1}$ on $L_{d}^{2}(\mathbf{F})$ determines a representation

$$
\sigma: C^{*} G_{1} \rightarrow \mathcal{L}\left(L_{d}^{2}(\mathbf{F})\right) .
$$

With a grading on $L_{d}^{2}(\mathbf{F})$ arising from the grading on $\mathbf{F}$,

$$
\left(L_{d}^{2}(\mathbf{F}), J_{\circ}\left(1+J^{2}\right)^{-1 / 2}, \sigma\right)
$$

defines a Kasparov $\left(C^{*} G_{1}, \mathbb{C}\right)$-bimodule.

Proof. In this proof all operators act on $L_{d}^{2}(\mathbf{F})$. Because $J \circ\left(1+J^{2}\right)^{-1 / 2}$ is a self-adjoint operator that commutes with the action of $G_{1}$, it suffices to show that for each $a \in C_{c}^{\infty}\left(G_{1}\right) \subset C^{*} G_{1}$

$$
\left(\left(\Im \circ\left(1+J^{2}\right)^{-1 / 2}\right)^{2}-I\right) \circ \sigma(a)
$$

is compact. We have

$$
\left(J \circ\left(1+J^{2}\right)^{-1 / 2}\right)^{2}-I=-\left(1+J^{2}\right)^{-1} .
$$


By Corollary 2.5, $\left(\rho\left(1-\Delta_{1}\right)+1+J^{2}\right)^{-1} \circ \sigma(a)$ is compact. It suffices to show that

$$
\left(\left(1+J^{2}\right)^{-1}-\left(\rho\left(1-\Delta_{1}\right)+1+J^{2}\right)^{-1}\right) \circ \sigma(a)
$$

is compact. The operator in (3.9) equals

$$
\left(1+J^{2}\right)^{-1} \circ\left(\rho\left(1-\Delta_{1}\right)+1+J^{2}\right)^{-1} \circ \rho\left(1-\Delta_{1}\right) \circ \sigma(a) .
$$

By [War, Prop. 4.4.1.2], $\rho\left(1-\Delta_{1}\right) \circ \sigma(a)$ is bounded. By Corollary 2.5, (3.10) is compact.

COROLlaRY 3.11. Give kernel( $\left(\left.J\right|_{L_{d}^{2}(\mathbf{F})}\right)$ the grading and action of $C^{*} G_{1}$ inherited from $L_{d}^{2}(\mathbf{F})$. Then $\left(\operatorname{kernel}\left(\left.\mathfrak{J}\right|_{L_{d}^{2}(\mathbf{F})}\right), 0, \sigma\right)$ is a Kasparov $\left(C^{*} G_{1}, \mathbb{C}\right)$ bimodule representing the same class in $K K\left(C^{*} G_{1}, \mathbb{C}\right)$ as the bimodule of Theorem 3.8.

Proof. $\mathfrak{J}$ commutes with $\sigma\left(C^{*} G_{1}\right)$.

\section{The Kasparov Product}

A Dirac operator $D_{V}$ on $K_{1} \backslash G_{1}$ defines an element $\left[\left(E_{\tilde{\mathbf{V}}}, D_{V} \circ\left(1+D_{V}^{2}\right)^{-1 / 2}\right)\right]$ of $K K\left(\mathbb{C}, C^{*} G_{1}\right)$ (see [K2]). Here $K_{1}$ is the maximal compact subgroup of $G_{1}$, and $E_{\tilde{\mathbf{v}}}$ is the Hilbert $C^{*} G_{1}$-module defined by sections of a homogeneous vector bundle $\tilde{\mathbf{V}} \rightarrow K_{1} \backslash G_{1}$ associated with a representation of $K_{1}$ on a vector space $V$. This vector bundle is the tensor product of the spinor bundle with an auxiliary bundle, and $E_{\tilde{\mathbf{v}}}$ gets a grading from the grading of the spinors. (We are assuming now that $K_{1} \backslash G_{1}$ is even-dimensional and has an invariant spin structure.) Let $\mathbf{F}$ and $J$ be as in Section 3 . In this section we compute the Kasparov product

$$
\left[\left(E_{\tilde{\mathbf{V}}}, D_{V} \circ\left(1+D_{V}^{2}\right)^{-1 / 2}\right)\right] \otimes_{C^{*} G_{1}}\left[\left(L_{d}^{2}(\mathbf{F}), J \circ\left(1+J^{2}\right)^{-1 / 2}, \sigma\right)\right]
$$

which lies in $K K(\mathbb{C}, \mathbb{C})$.

Notation 4.1. Let $\mathbf{V}$ denote the product vector bundle $V \times(H \backslash G / \Gamma)$.

Proposition 4.2. The map

given by

$$
Q: C_{c}^{\infty}\left(G_{1}, V\right)^{K_{1}} \otimes L^{2}(\mathbf{F}) \rightarrow L^{2}(\mathbf{V} \otimes \mathbf{F})^{K_{1}}
$$

$$
Q(f \otimes \xi)=\int_{G_{1}} f(g) \otimes(g \cdot \xi)(x) d g
$$

extends to define an isomorphism

$$
Q: E_{\tilde{\mathbf{V}}} \otimes_{C^{*} G_{1}} L^{2}(\mathbf{F}) \cong L^{2}(\mathbf{V} \otimes \mathbf{F})^{K_{1}}
$$

If we let $K_{1} \backslash(\mathbf{V} \otimes \mathbf{F})$ denote the quotient bundle over $\left(K_{1} \times H\right) \backslash G / \Gamma$, then

$$
\begin{aligned}
L^{2}(\mathbf{V} \otimes \mathbf{F})^{K_{1}} & \cong L^{2}\left(K_{1} \backslash(\mathbf{V} \otimes \mathbf{F})\right) \\
& \cong\left(V \otimes F \otimes L^{2}(G / \Gamma)\right)^{K_{1} \times H}
\end{aligned}
$$


Each of these isomorphisms respects the splittings into discrete and continuous summands, which we denote as usual by subscripts $d$ and c. For instance, we have

$$
\begin{aligned}
L_{d}^{2}(\mathbf{V} \otimes \mathbf{F})^{K_{1}} & \cong L_{d}^{2}\left(K_{1} \backslash(\mathbf{V} \otimes \mathbf{F})\right) \\
& \cong\left(V \otimes F \otimes L_{d}^{2}(G / \Gamma)\right)^{K_{1} \times H} .
\end{aligned}
$$

Proof. The proof is analogous to the proofs of similar statements in [FH1] and [FH2].

REMARK 4.3. Because $G_{1} \subset G$ and because $F$ is finite-dimensional, the set of $C^{\infty}$ vectors for the action of $G_{1}$ on $L^{2}(\mathbf{F})$ contains $\left(F \otimes L^{2}(G / \Gamma)_{\infty}\right)^{H}$. Thus the image of $C_{c}^{\infty}\left(G_{1}, V\right)^{K_{1}} \otimes\left(F \otimes L^{2}(G / \Gamma)_{\infty}\right)^{H}$ under $Q$ is contained in the tensor product of $V$ with the set of $C^{\infty}$ vectors for the action of $G_{1}$ on $L^{2}(\mathbf{F})$.

Definition 4.4. Let $Y \in \mathfrak{g}_{1}$, the Lie algebra of $G_{1}$. Recall that there is a linear map $\operatorname{cl}(Y): V \rightarrow V$ given by the tensor product of Clifford multiplication by $Y$ on the spinors and the identity on the auxiliary factor. Define a vector bundle map

by

$$
c(Y): \mathbf{V} \otimes \mathbf{F} \rightarrow \mathbf{V} \otimes \mathbf{F}
$$

$$
c(Y)_{x}=\operatorname{cl}(Y) \otimes \operatorname{Id}_{\mathbf{F}_{x}} \text {. }
$$

Definition 4.5. Let $Y \in \mathrm{g}_{1}$. Define a differential operator $d(Y)$ on the tensor product of $V$ with the set of $C^{\infty}$ vectors for the action of $G_{1}$ on $L^{2}(\mathbf{F})$ by

$$
[d(Y) \eta](x)=\left.c(Y) \frac{d}{d t}\right|_{t=0} \exp (t Y) \eta\left(\exp (t Y)^{-1} x\right) .
$$

DEFINITION 4.6. Give $g_{1}$ an inner product that is invariant under the adjoint action of $K_{1}$. Let $k_{1}$ be the Lie algebra of $K_{1}$, and let $p_{1}$ be the orthogonal complement of $k_{1}$ in $g_{1}$. Let $\left\{Y_{1}, \ldots, Y_{n}\right\}$ be an orthonormal basis for $p_{1}$. Let

$$
\underline{D}_{V}=\sum_{i=1}^{n} d\left(Y_{i}\right) \text {. }
$$

Lemma 4.8. $\mathfrak{D}_{V}$ is $K_{1}$-invariant; thus it defines a differential operator on $\left(K_{1} \times H\right) \backslash G / \Gamma$. This operator acts on $\left(V \otimes\left\{C^{\infty}\right.\right.$ vectors for the action of $G_{1}$ on $\left.\left.L^{2}(\mathbf{F})\right\}\right)^{K_{1}}$. It is the descended version of a $G$-invariant differential operator on $\left(K_{1} \times H\right) \backslash G$.

Proof. The proof is a computation analogous to that commonly used to show that a formula analogous to (4.7) defines a Dirac operator on $K_{1} \backslash G_{1}$.

Notation 4.9. Let $\mathfrak{D}_{V}$ denote the operator on $\left(K_{1} \times H\right) \backslash G / \Gamma$ described in the preceding lemma.

LEMma 4.10. For $f \in C_{c}^{\infty}\left(G_{1}, V\right)^{K_{1}}$ and $\xi \in\left(F \otimes L^{2}(G / \Gamma)_{\infty}\right)^{H}$, 


$$
\mathscr{D}_{V}(Q(f \otimes \xi))=Q\left(D_{V}(f) \otimes \xi\right) .
$$

Proof. The proof is a calculation involving a change of variable in the integral defining $Q$.

REMARK 4.11. $]$ defines another operator $\underline{\mathfrak{J}}_{V}$ on $V \otimes\left(F \otimes L^{2}(G / \Gamma)_{\infty}\right)^{H}$ as follows. For $v \in V$ of pure degree and for $\xi \in\left(F \otimes L^{2}(G / \Gamma)_{\infty}\right)^{H}$,

$$
\underline{\Im}_{V}(v \otimes \xi)=(-1)^{\operatorname{deg}(v)} v \otimes J(\xi) .
$$

Because $\mathfrak{I}$ is $K_{1}$-invariant, $\underline{\jmath}_{V}$ defines a differential operator on $\left(K_{1} \times H\right) \backslash$ $G / \Gamma$ that acts on $\left(V \otimes F \otimes L^{2}(G / \Gamma)_{\infty}\right)^{K_{1} \times H}$. The operator is the descended version of a $G$-invariant differential operator on $\left(K_{1} \times H\right) \backslash G$.

Notation 4.12. Let $I_{V}$ denote the operator on $\left(K_{1} \times H\right) \backslash G / \Gamma$ described in the preceding remark.

Notation 4.13. Define an operator $\mathcal{P}_{V}$ on $\left(V \otimes F \otimes L^{2}(G / \Gamma)_{\infty}\right)^{K_{1} \times H}$ by

$$
\mathcal{P}_{V}=\mathscr{D}_{V}+\mathfrak{J}_{V}
$$

Proposition 4.14. $\quad \mathcal{P}_{V}$ is a first-order elliptic operator and $\mathcal{P}_{V}^{2}$ a secondorder elliptic operator on $\left(K_{1} \times H\right) \backslash G / \Gamma$. Each operator is descended from a G-invariant elliptic operator on $\left(K_{1} \times H\right) \backslash G$.

Proof. Use ellipticity of $D$ on $K_{1} \backslash G_{1}$ and transversal ellipticity of $T$ to calculate with principal symbols.

Proposition 4.15. $\quad \mathcal{P}_{V}$ and $\odot_{V}^{2}$, with domain $\left(V \otimes F \otimes L^{2}(G / \Gamma)_{\infty}\right)^{K_{1} \times H}$, are symmetric and thus essentially self-adjoint.

Proof. This follows from the symmetry of $\mathcal{D}$ and of $\mathfrak{I}$ and from Proposition 2.6.

LEMMA 4.16. $\mathfrak{D}_{V} \circ \mathfrak{J}_{V}=-\mathfrak{J}_{V} \circ \mathfrak{D}_{V}$ on $\left(V \otimes F \otimes L_{d}^{2}(G / \Gamma)\right)^{K_{1} \times H}$.

Proof. The proof is a computation using the definitions of the operators and the $G_{1}$-invariance of $\mathfrak{J}$.

Proposition 4.17. $\mathfrak{D}_{V}$ and $\Im_{V}$, with domain $\left(V \otimes F \otimes L_{d}^{2}(G / \Gamma)_{\infty}\right)^{K_{1} \times H}$, are essentially self-adjoint operators on $\left(V \otimes F \otimes L_{d}^{2}(G / \Gamma)\right)^{K_{1} \times H}$.

Proof. $\mathfrak{D}_{V}$ and $I_{V}$ satisfy the assumptions of Proposition 2.10 with respect to the self-adjoint elliptic operator $1+\odot_{V}^{2}$.

Notation 4.18. We use notations such as $\mathcal{P}_{V}, \odot_{V}^{2}, \mathscr{D}_{V}$, and $\mathfrak{J}_{V}$ to refer to the closures of the discrete blocks of the operators of the same names.

REMARK 4.19. Let $P$ be a self-adjoint operator on a Hilbert space. We will use the identity introduced in a similar context by [BJ]: 


$$
\left(1+P^{2}\right)^{-1 / 2}=\frac{1}{\pi} \int_{0}^{\infty} \lambda^{-1 / 2}\left(1+P^{2}+\lambda\right)^{-1} d \lambda .
$$

The right side is interpreted as the norm limit of functional calculus expressions arising from Riemann sums for approximating proper integrals.

LEMma 4.20. Suppose $f \in C^{\infty}\left(G_{1}, V\right)^{K_{1}}$ is such that $\left(1+D_{V}^{2}\right) f \in C_{c}^{\infty}\left(G_{1}, V\right)^{K_{1}}$. Then $f \in E_{\tilde{\mathbf{V}}}$. Assume $\xi \in\left(F \otimes L_{d}^{2}(G / \Gamma)_{\infty}\right)^{H}$. Then

$$
Q\left(\left(1+D_{V}^{2}\right) f \otimes \xi\right)=\left(1+D_{V}^{2}\right)(Q(f \otimes \xi)) .
$$

Proof. Because $1+D_{V}^{2}$ is a closed operator, this lemma is a consequence of Lemma 4.10 and the proof of Theorem 2 of [K2].

Lemma 4.21. On the discrete blocks $Q \circ\left(\left(1+D_{V}^{2}\right)^{-1 / 2} \otimes 1\right)=\left(1+D_{V}^{2}\right)^{-1 / 2}$.

Proof. Because the operators in question are bounded, it suffices to establish the equality for $f \otimes \xi \in C_{c}^{\infty}\left(G_{1}, V\right)^{K_{1}} \otimes\left(F \otimes L_{d}^{2}(G / \Gamma)_{\infty}\right)^{H}$. Remark 4.19 enables us to apply Lemma 4.20 to finish the proof.

Lemma 4.22. On the discrete blocks

$$
Q \circ\left(D_{V} \circ\left(1+D_{V}^{2}\right)^{-1 / 2} \otimes 1\right)=\mathscr{D}_{V} \circ\left(1+D_{V}^{2}\right)^{-1 / 2} .
$$

Proof. Analyze $Q \circ\left(\left(1+D_{V}^{2}\right)^{-1 / 2} D_{V} \otimes 1\right)$ and $\left(1+D_{V}^{2}\right)^{-1 / 2} \mathscr{D}_{V}$ as in the proof of the preceding lemma.

THEOREM 4.23.

$$
\begin{aligned}
& {\left[\left(E_{\tilde{\mathbf{V}}}, D_{V^{\circ}}\left(1+D_{V}^{2}\right)^{-1 / 2}\right)\right] \otimes_{C^{*} G_{1}}\left[\left(L_{d}^{2}(\mathbf{F}), J^{\circ}\left(1+J^{2}\right)^{-1 / 2}, \sigma\right)\right]} \\
& \left.\quad=\left[\left(V \otimes F \otimes L_{d}^{2}(G / \Gamma)\right)^{K_{1} \times H}, \mathcal{P}_{V^{\circ}}\left(1+\mathcal{\odot}_{V}^{2}\right)^{-1 / 2}\right)\right] \in K K(\mathbb{C}, \mathbb{C}) .
\end{aligned}
$$

Proof. In the rest of this section all operators are restricted to discrete blocks. We use the connection approach (see either [Sk] or [Bl]) to compute Kasparov products. Proposition 4.2 identifies the module appearing in the product. $\left(\left(V \otimes F \otimes L_{d}^{2}(G / \Gamma)\right)^{K_{1} \times H}, \mathcal{P}_{V^{\circ}}\left(1+\mathcal{P}_{V}^{2}\right)^{-1 / 2}\right)$ is a Kasparov $(\mathbb{C}, \mathbb{C})$ bimodule because $\mathcal{P}_{V^{\circ}}\left(1+\mathcal{Q}_{V}^{2}\right)^{-1 / 2}$ is self-adjoint and because

$$
\left(\mathcal{P}_{V} \circ\left(1+\mathcal{P}_{V}^{2}\right)^{-1 / 2}\right)^{2}-I=-\left(1+\mathcal{P}_{V}^{2}\right)^{-1},
$$

to which Corollary 2.5 applies.

Proposition 4.25, which establishes the positivity condition of the definition of Kasparov product, and Proposition 4.26, which establishes the connection condition, finish the proof of this theorem.

NotATion 4.24. Let $U_{\alpha}$ denote the eigenspace for $\mathcal{P}_{V}^{2}$ associated with eigenvalue $\alpha$. Let $U$ denote the algebraic direct sum

$$
U=\oplus_{\alpha} U_{\alpha} \text {. }
$$

Proposition 4.25. $\left[D_{V^{\circ}}\left(1+D_{V}^{2}\right)^{-1 / 2} \otimes 1, \mathcal{P}_{V^{\circ}}\left(1+\mathcal{P}_{V}^{2}\right)^{-1 / 2}\right] \geq 0$ modulo compact operators. 
Proof. Note that by slight abuse of notation we have ommitted $Q$. Recall that commutator brackets denote graded commutators.

It suffices to establish nonnegativity of this bounded operator on the dense subset $U$. The following formal manipulations are justified by restriction to $U$ :

$$
\begin{aligned}
& {\left[D_{V^{\circ}}\left(1+D_{V}^{2}\right)^{-1 / 2} \otimes 1, \mathcal{P}_{V^{\circ}}\left(1+\mathcal{P}_{V}^{2}\right)^{-1 / 2}\right]} \\
& =\left[\mathscr{D}_{V} \circ\left(1+\mathscr{D}_{V}^{2}\right)^{-1 / 2}, \mathfrak{D}_{V}\right]\left(1+\mathcal{P}_{V}^{2}\right)^{-1 / 2} \\
& =\left(1+\mathscr{D}_{V}^{2}\right)^{-1 / 2} 2 D_{V}^{2}\left(1+\mathcal{P}_{V}^{2}\right)^{-1 / 2} \text {. }
\end{aligned}
$$

For each $u \in U,\left\langle\left(1+D_{V}^{2}\right)^{-1 / 2} 2 D_{V}^{2}\left(1+\odot_{V}^{2}\right)^{-1 / 2} u, u\right\rangle \geq 0$.

The commutator manipulations omitted in the above are analogous to the ones that appear in full detail in the proof of Proposition 3.27 of [FH2].

Proposition 4.26. For $f \in E_{\tilde{\mathrm{V}}}$, let $Q_{f}$ denote the map

$$
L_{d}^{2}(\mathbf{F}) \rightarrow L_{d}^{2}\left(K_{1} \backslash(\mathbf{V} \otimes \mathbf{F})\right)
$$

defined by $Q_{f}(\xi)=Q(f \otimes \xi)$. Then for $f \in C_{c}^{\infty}\left(G_{1}, V\right)^{K_{1}}$ of pure degree,

$$
Q_{f} \circ \Im \circ\left(1+\Im^{2}\right)^{-1 / 2}-(-1)^{\operatorname{deg}(f)} \mathcal{P}_{V} \circ\left(1+\odot_{V}^{2}\right)^{-1 / 2} \circ Q_{f}
$$

is compact. Because the ideal of compact operators is norm-closed and because the adjoint of a compact operator is compact, this result establishes the connection condition for the Kasparov product.

Proof.

$$
\begin{aligned}
& Q_{f} \circ J^{\circ}\left(1+J^{2}\right)^{-1 / 2}-(-1)^{\operatorname{deg}(f)} \mathcal{P}_{V} \circ\left(1+\mathcal{P}_{V}^{2}\right)^{-1 / 2} \circ Q_{f} \\
& =(-1)^{\operatorname{deg}(f)}\left(J_{V} \circ\left(1+J_{V}^{2}\right)^{-1 / 2}-\mathcal{P}_{V} \circ\left(1+\odot_{V}^{2}\right)^{-1 / 2}\right) \cdot Q_{f} \\
& =(-1)^{\operatorname{deg}(f)} J_{V}\left(\left(1+J_{V}^{2}\right)^{-1 / 2}-\left(1+\mathcal{P}_{V}^{2}\right)^{-1 / 2}\right) \cdot Q_{f}-\left(1+\mathcal{P}_{V}^{2}\right)^{-1 / 2} \circ D_{V} \circ Q_{f} .
\end{aligned}
$$

The second term after the last equals sign is compact since $D_{V}{ }^{\circ} Q_{f}$ is bounded by [War, Prop. 4.4.1.2] and $\left(1+\mathcal{P}_{V}^{2}\right)^{-1 / 2}$ is compact by Corollary 2.5. The same references establish the boundedness of $\mathfrak{D}_{V}^{2} Q_{f}$ and the compactness of $\left(1+\odot_{V}^{2}\right)^{-1 / 2}$. Therefore, the following calculation establishes the compactness of the first term after the last equals sign in the preceding display:

$$
\begin{aligned}
\Im_{V}\left(\left(1+\Im_{V}^{2}\right)^{-1 / 2}-\left(1+\odot_{V}^{2}\right)^{-1 / 2}\right) \circ Q_{f} \\
\quad=\frac{1}{\pi} \int_{0}^{\infty} \lambda^{-1 / 2} \Im_{V}\left(\left(1+\Im_{V}^{2}+\lambda\right)^{-1}-\left(1+\odot_{V}^{2}+\lambda\right)^{-1}\right) Q_{f} d \lambda \\
=\frac{1}{\pi} \int_{0}^{\infty} \lambda^{-1 / 2} \Im_{V}\left(1+\Im_{V}^{2}+\lambda\right)^{-1}\left(1+\odot_{V}^{2}+\lambda\right)^{-1} D_{V}^{2} Q_{f} d \lambda .
\end{aligned}
$$

REMARK 4.27. The standard identification of $K K(\mathbb{C}, \mathbb{C})$ with $\mathbb{Z}$ takes the class represented by the Kasparov bimodule of Theorem 4.23 to the index of the lower left-hand entry of the discrete block of $\boldsymbol{P}_{V}$. 


\section{The Product Revisited: Conclusion}

In the preceding section we calculated the Kasparov product of an element of $K K\left(\mathbb{C}, C^{*} G_{1}\right)$ defined by a Dirac operator on $K_{1} \backslash G_{1}$ and an element of $K K\left(C^{*} G_{1}, \mathbb{C}\right)$ defined by a transversally elliptic operator. The result, interpreted as an integer via the standard identification $K K(\mathbb{C}, \mathbb{C}) \cong \mathbb{Z}$, is the index of the discrete block of an explicitly described elliptic operator on a locally homogeneous space.

Corollary 3.11 identifies a different cycle-one representing the class in $K K\left(C^{*} G_{1}, \mathbb{C}\right)$ used above. If we use this different cycle to calculate the same Kasparov product, we get a different interpretation of the product. This interpretation identifies the resulting integer as a linear combination of multiplicities of irreducible $G_{1}$-representations in the kernel of the operator $\mathfrak{J}$ of Theorem 3.8. The irreducible $G_{1}$-representations whose multiplicities appear are determined by the Dirac operator $D_{V}$. This approach is completely independent of the nature of the space on which the transversally elliptic operator lives. Thus the calculations in the present noncompact setting are exactly the same as those done in the compact case in [FH2]. In this paper we restrict ourselves to noting their most interesting consequences.

Notation 5.1. Let $\beta$ be an irreducible unitary $G_{1}$-representation. Let $\exists$ be the operator on $L_{d}^{2}(\mathbf{F})$ of Theorem 3.8. Denote the even and odd parts of the kernel of $\Im$ by kernel $(\Im)^{0}$ and $\operatorname{kernel}(\zeta)^{1}$, respectively. Define

$$
m_{T}(\beta)=\sum_{i=0,1}(-1)^{i}\left(\text { multiplicity of } \beta \text { in } \operatorname{kernel}(\Im)^{i}\right) .
$$

THEOREM 5.2. Let $\mathfrak{J}$ be the operator constructed from a transversally elliptic operator as in Section 3. Let $\beta$ be a discrete series representation of $G_{1}$ that satisfies the positivity condition of [W1] and [W2]. Let $D_{V(\beta)}$ be the Dirac operator on $K_{1} \backslash G_{1}$ that realizes this discrete series representation. Let

$$
\mathcal{P}_{V(\beta)}=\mathfrak{D}_{V(\beta)}+\mathfrak{J}_{V(\beta)}
$$

be the operator constructed in Section 4. Let index $\left(\mathcal{P}_{V(\beta)}\right)$ be the index described in Remark 4.27. Then

$$
m_{T}(\beta)=\operatorname{index}\left(\odot_{V(\beta)}\right) .
$$

Proof. Each of these numbers is the image in $\mathbb{Z}$ of the same Kasparov product.

\section{References}

[At] M. Atiyah, Elliptic operators and compact groups, Lecture Notes in Math., 401, Springer, New York, 1974.

[AS] M. Atiyah and W. Schmid, A geometric construction of the discrete series for semisimple Lie groups, Invent. Math. 42 (1977), 1-62. 
[BJ] S. Baaj and P. Julg, Théorie bivariante de Kasparov et opérateurs non bornés dans les $C^{*}$-modules Hilbertiens, C. R. Acad. Sci. Paris Sér. I Math. 296 (1983), 875-878.

[Bl] B. Blackadar, K-theory for operator algebras, Math. Sci. Res. Inst. Publ., 5, Springer, New York, 1986.

[Bo] A. Borel, Compact Clifford-Klein forms of symmetric spaces, Topology 2 (1963), 111-122.

[BG] A. Borel and H. Garland, Laplacian and the discrete spectrum of an arithmetic group, Amer. J. Math. 105 (1983), 309-335.

[BH] A. Borel and Harish-Chandra, Arithmetic subgroups of algebraic groups, Ann. of Math. (2) 75 (1962), 485-535.

[Co] A. Connes, Non-commutative differential geometry, Publ. Math. IHES 62 (1985), 41-144.

[CM] A. Connes and H. Moscovici, The $L^{2}$-index theorem for homogeneous spaces of Lie groups, Ann. of Math. (2) 115 (1982), 291-330.

[CS] A. Connes and G. Skandalis, The longitudinal index theorem for foliations, Publ. Res. Inst. Math. Sci. 20 (1984), 1139-1183.

[FH1] J. Fox and P. Haskell, Index theory on locally homogeneous spaces, $K$-Theory 4 (1991), 547-568.

[FH2] - The index of transversally elliptic operators for locally free actions, Pacific J. Math. (to appear).

[HS] M. Hilsum and G. Skandalis, Morphismes K-orientés d'espaces de feuilles et fonctorialité en théorie de Kasparov, Ann. Sci. École Norm. Sup. (4) 20 (1987), 325-390.

[K1] G. Kasparov, Equivariant KK-theory and the Novikov conjecture, Invent. Math. 91 (1988), 147-201.

[K2] - An index for invariant elliptic operators, $K$-theory, and representations of Lie groups, Soviet Math. Dokl. 27 (1983), 105-109.

[K3] - The operator $K$-functor and extensions of $C^{*}$-algebras, Math. USSRIzv. 16 (1981), 513-572.

[Mo] H. Moscovici, $L^{2}$-index of elliptic operators on locally symmetric spaces of finite volume, Operator algebras and $K$-theory (R. Douglas, $\mathrm{C}$. Schochet, eds.), Contemp. Math., 10, pp. 129-137, Amer. Math. Soc., Providence, RI, 1982.

[NS] E. Nelson and F. Stinespring, Representation of elliptic operators in an enveloping algebra, Amer. J. Math. 81 (1959), 547-560.

[NZ] A. Nestke and F. Zickermann, The index of transversally elliptic complexes, Rend. Circ. Mat. Palermo (2) Suppl. 9 (1985), 165-175.

[Pa] R. Parthasarathy, Dirac operator and the discrete series, Ann. of Math. (2) 96 (1972), 1-30.

[Pe] M. Penington, $K$-theory and $C^{*}$-algebras of Lie groups and foliations, D.Phil. thesis, Oxford Univ., 1983.

[RS] M. Reed and B. Simon, Methods of modern mathematical physics. II. Fourier analysis, self-adjointness, Academic Press, New York, 1975.

[Si] I. Singer, Future extensions of index theory and elliptic operators, Prospects in mathematics, Ann. of Math. Stud., 70, Princeton Univ. Press, Princeton, NJ, 1971.

[Sk] G. Skandalis, Some remarks on Kasparov theory, J. Funct. Anal. 56 (1984), 337-347. 
[Ta] M. Taylor, Pseudodifferential operators, Princeton Univ. Press, Princeton, NJ, 1981.

[Ve] M. Vergne, Sur l'indice des opérateurs transversalement elliptiques, C. R. Acad. Sci. Paris Sér. I Math. 310 (1990), 329-332.

[War] G. Warner, Harmonic analysis on semi-simple Lie groups I and II, Springer, New York, 1972.

[Was] A. Wasserman, A proof of the Connes-Kasparov conjecture for connected reductive Lie groups, C. R. Acad. Sci. Paris Sér. I Math. 304 (1987), 559-562.

[W1] F. Williams, Discrete series multiplicities in $L^{2}(\Gamma \backslash G)^{*}$, Amer. J. Math. 106 (1984), 137-148.

[W2] — Note on a theorem of H. Moscovici, J. Funct. Anal. 72 (1987), 2832.

[Zi] R. Zimmer, Ergodic theory and semisimple groups, Monographs Math., 81, Birkhäuser, Boston, 1984.

J. Fox

Department of Mathematics

University of Colorado

Boulder, CO 80309
P. Haskell

Department of Mathematics

Virginia Polytechnic Institute

\& State University

Blacksburg, VA 24061 